Elsevier Editorial System(tm) for Chemical Physics Letters

Manuscript Draft

Manuscript Number: CPLETT-06-1031R1

Title: Prediction of Increased Tunneling Current by Bond Length Stretch in Molecular Break Junctions

Article Type: Regular Article

Section/Category: Nanostructures and Materials

Keywords:

Corresponding Author: Prof Mike J Ford, PhD

Corresponding Author's Institution: University of Technology, Sydney

First Author: Rainer C Hoft, B.Sc. Hons

Order of Authors: Rainer C Hoft, B.Sc. Hons; Mike Ford, Ph.D.; Michael B Cortie, Ph.D.

Manuscript Region of Origin:

Abstract: We use ab-initio calculations of the tunneling current through a 1,4-phenylenedimethanethiol (XYL) molecule adsorbed between $\mathrm{Au}(111)$ electrodes to show that there are circumstances under which tunneling currents can be increased by bond stretching. The effect is dependent upon the geometry of the gold-sulfur bond and does not occur for the physisorbed thiol, that is, a thiol with the hydrogen atom still in place. However, we predict that when the hydrogen atom is removed, causing the sulfur atom to be strongly bound to the gold surface, stretching this bond away from equilibrium will actually increase the tunneling current. 
Rainer Hoft

Institute for Nanoscale Technology

University of Technology Sydney

PO Box 123 Broadway NSW 2007

Australia

18 May 2006

Professor David Clary

Editor

Chemical Physics Letters

Dear Prof Clary

Please find attached our manuscript for submission to Chemical Physics Letters entitled "Prediction of Increased Tunneling Current by Bond Length Stretch in Molecular Break Junctions".

The goal of this work was to test computationally the effect of different interface geometries on the molecular conductance of a simple electrode-molecule-electrode system. This is an area of active research due to the importance of understanding the effect of geometry variation in molecular break junction experiments that attempt to probe the conductance of individual molecules.

We reached the very surprising result that stretching and indeed breaking the gold-sulfur bond responsible for anchoring the molecule to the electrodes can significantly increase the conductance of the system. This counterintuitive result is supported by previous work; here we definitively show that it is a solid prediction which is not isolated to a particular system and not an artifact based on inherent difficulties of DFT to describe bond dissociation correctly. It is thus an important theoretical prediction in the area of molecular electronics where understanding of interface effects is critical to possible future device fabrication.

We believe this work is suitable for publication in Chemical Physics Letters and thank you for your consideration.

Yours sincerely, Rainer Hoft 


\section{Manuscipt title:}

Prediction of Increased Tunneling Current by Bond Length Stretch in Molecular Break Junctions

\section{Corrsponding Author:}

Mike Ford

Mike.ford@uts.edu.au

Associate Director

Institute for Nanoscale Technology

University of Technology, Sydney

PO Box 123, Broadway, NSW 2007

$\mathrm{Ph}: 61(0) 295147956$

Fax: 61(0)2 95148349

Mob: 0421070494

\section{Suggested reviewers:}

Prof. Kurt Stokbro

Nano-Science Center

H.C. Ørsted Instituttet

Universitetsparken 5, Bygning D

DK-2100 København Ø

ks@atomistix.com

Prof. Hong Guo

Department of Physics, McGill University

Rutherford Building, 3600 rue University

Montréal (Québec), PQ H3A 2T8, Canada

guo@physics.mcgill.ca

Prof. Mark Ratner

Department of Chemistry

Northwestern University

2145 Sheridan Road

Evanston, IL 60208-3113

ratner@chem.northwestern.edu 
Figure 1
Click here to download high resolution image

left

electrode device region

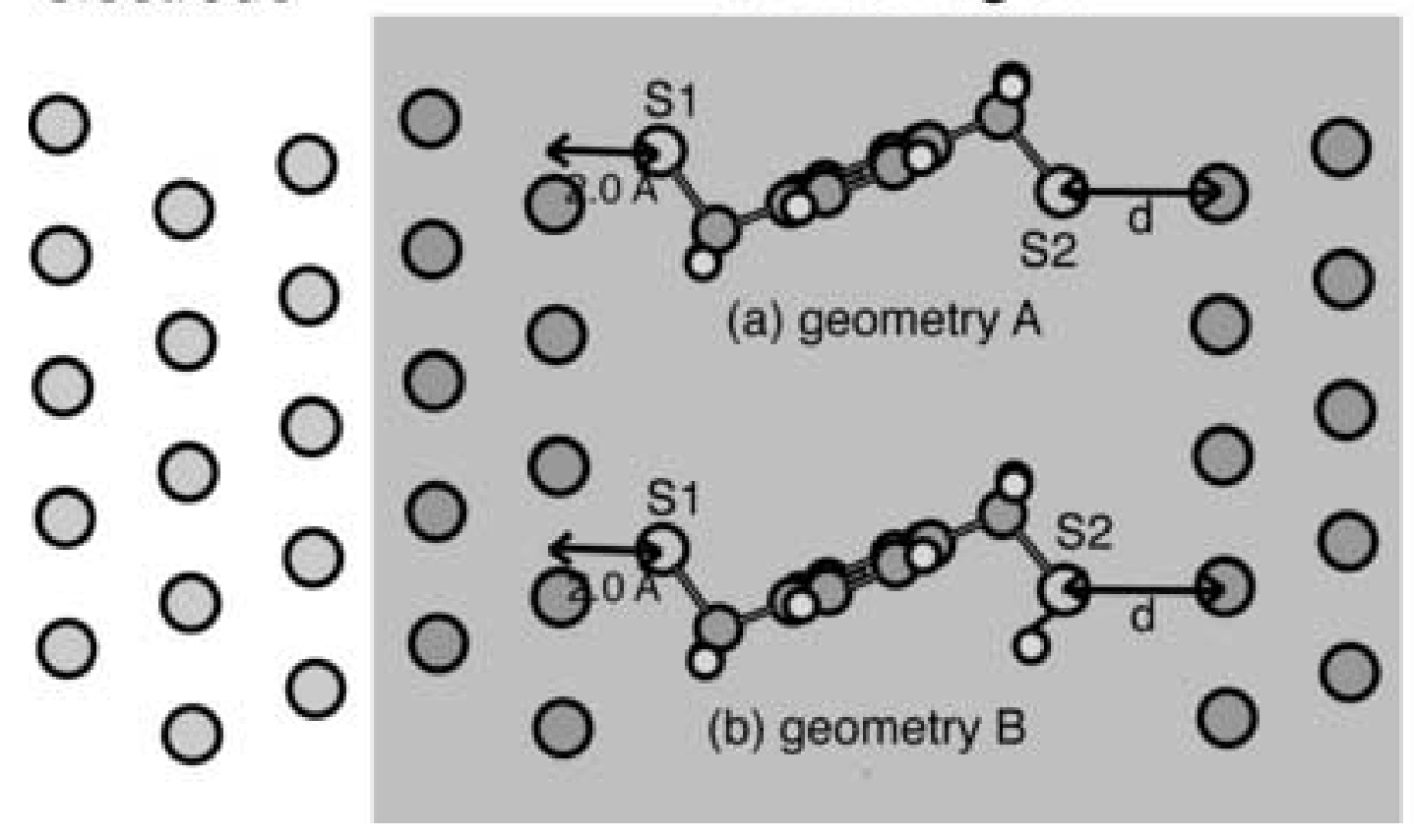

right electrode

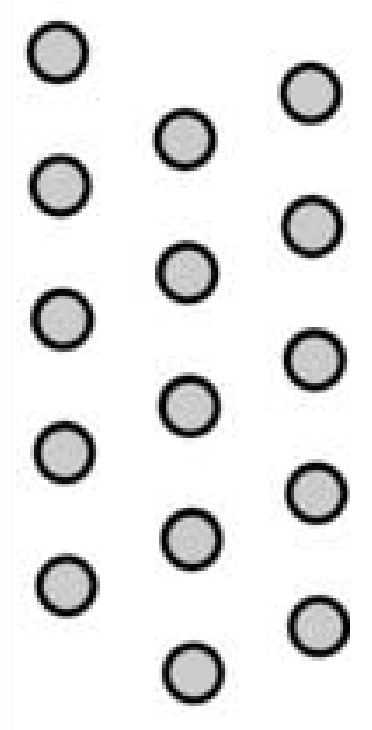


Figure 2
Click here to download high resolution image

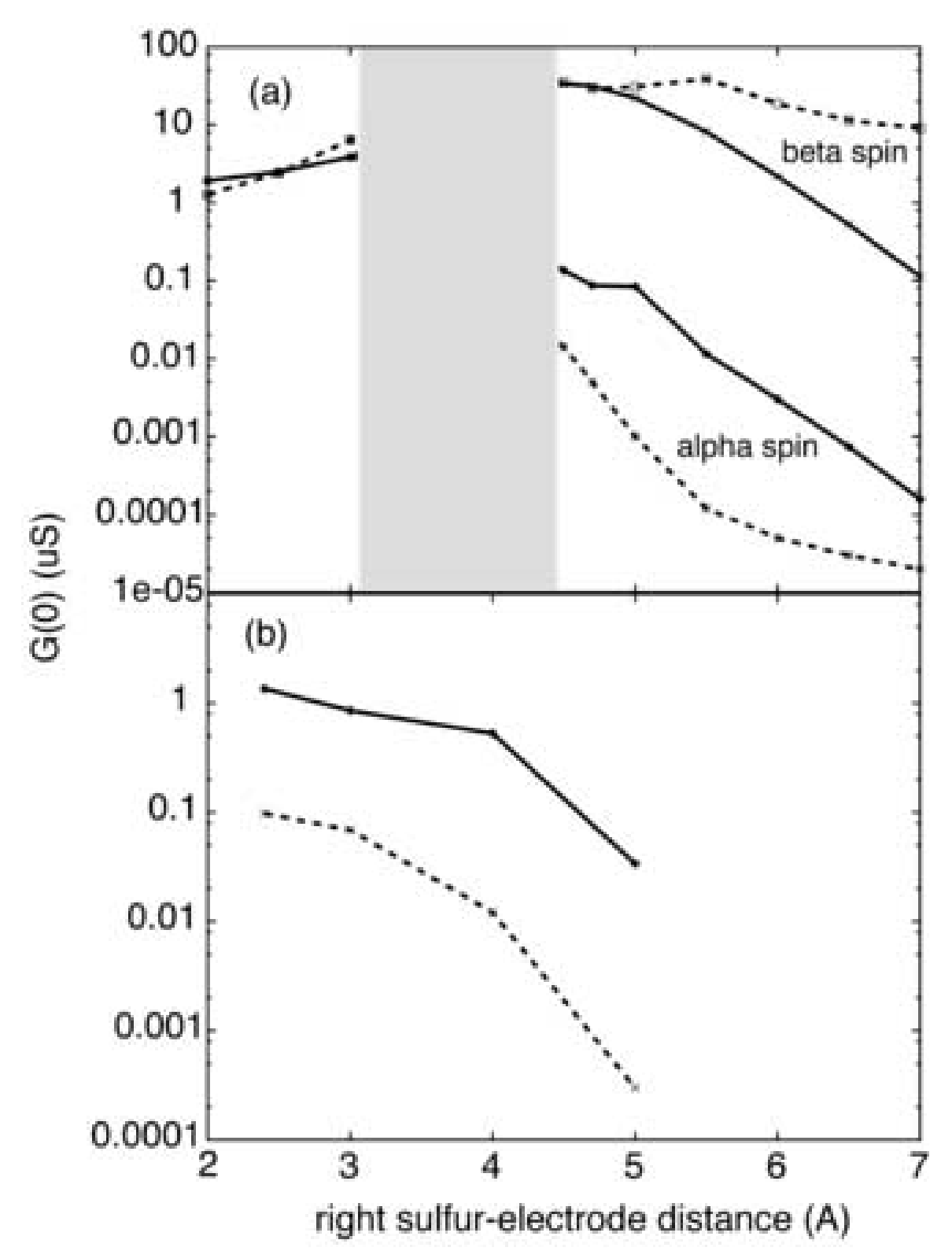




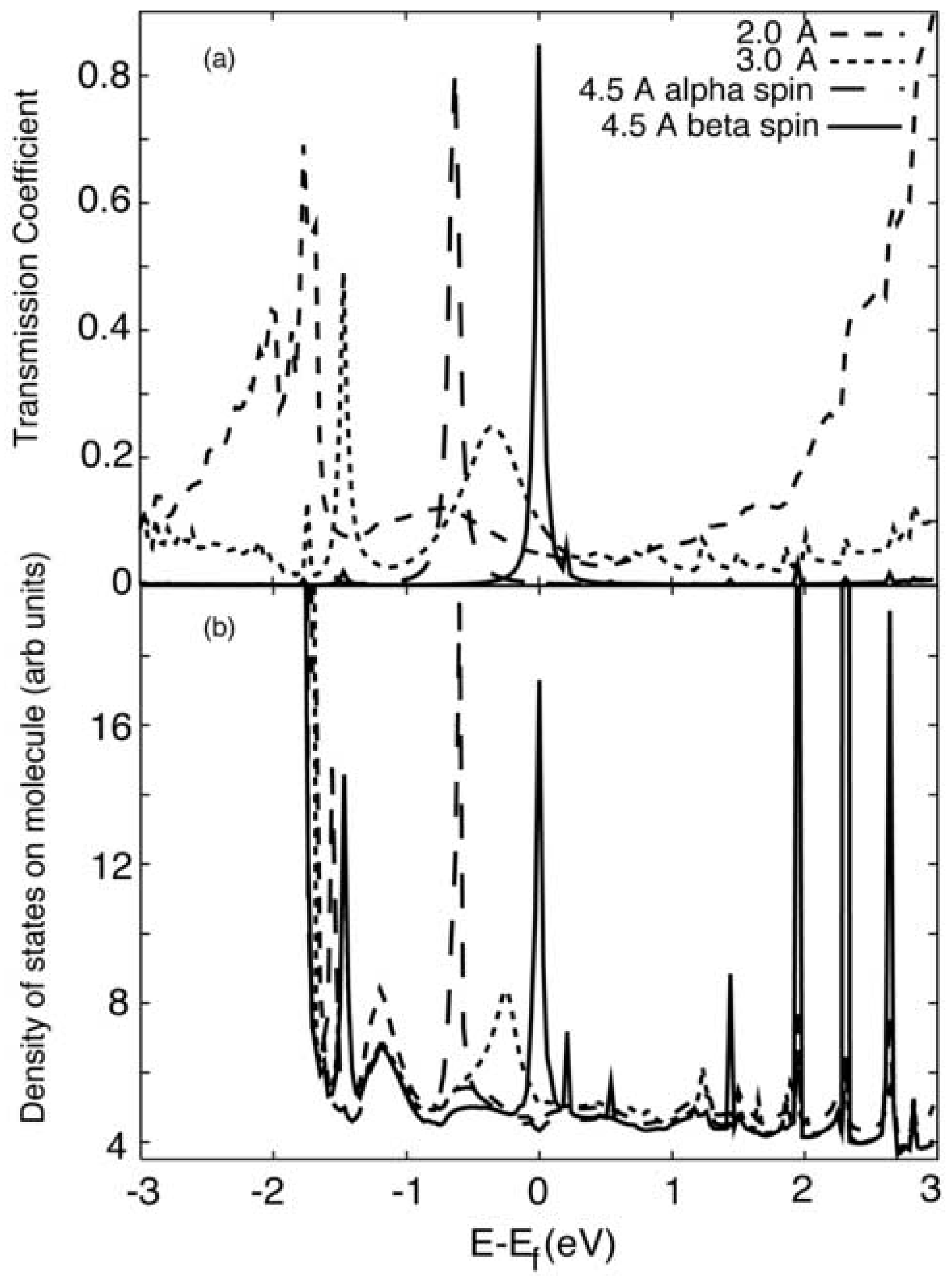


Figure 4
Click here to download high resolution image

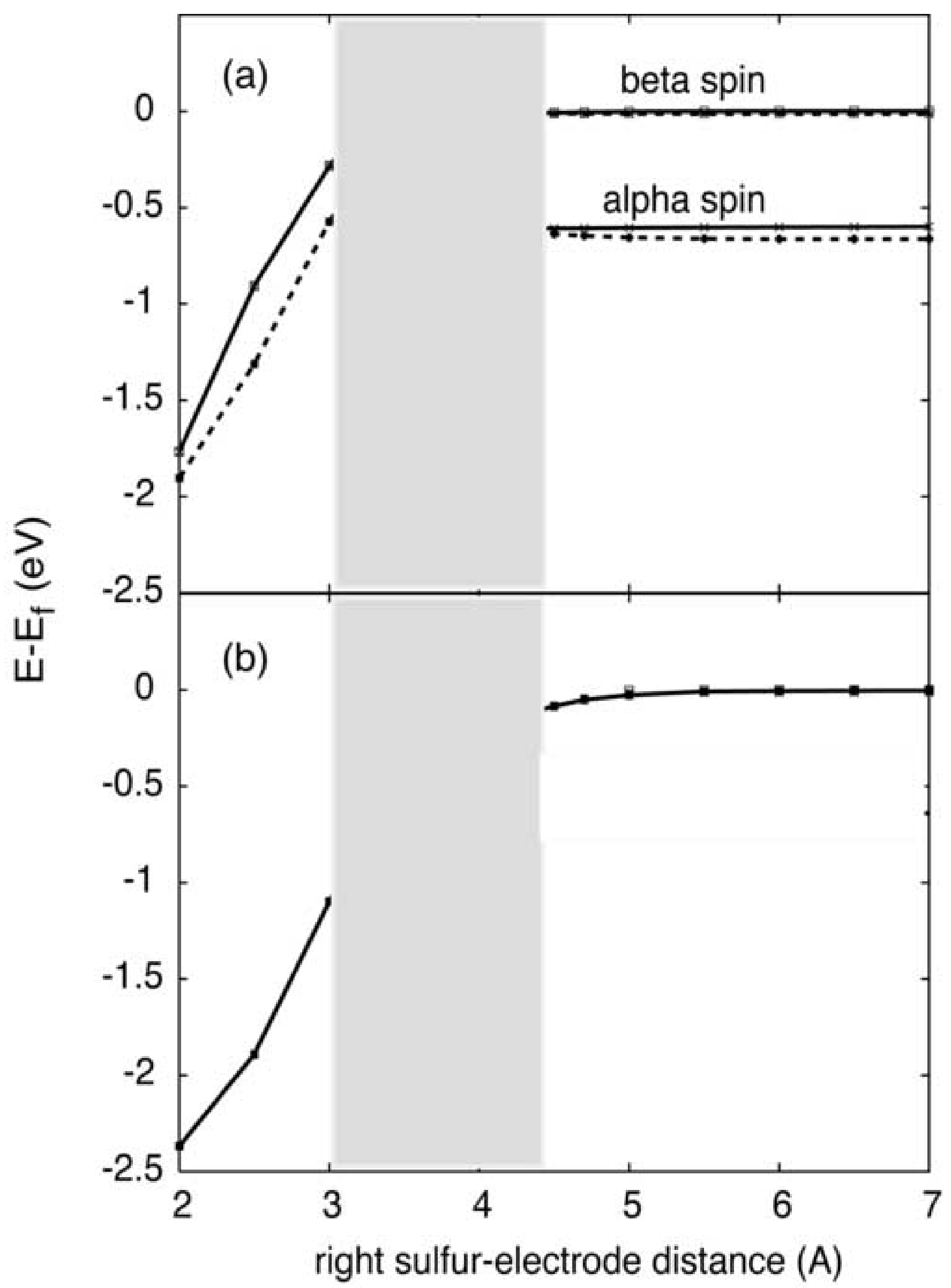



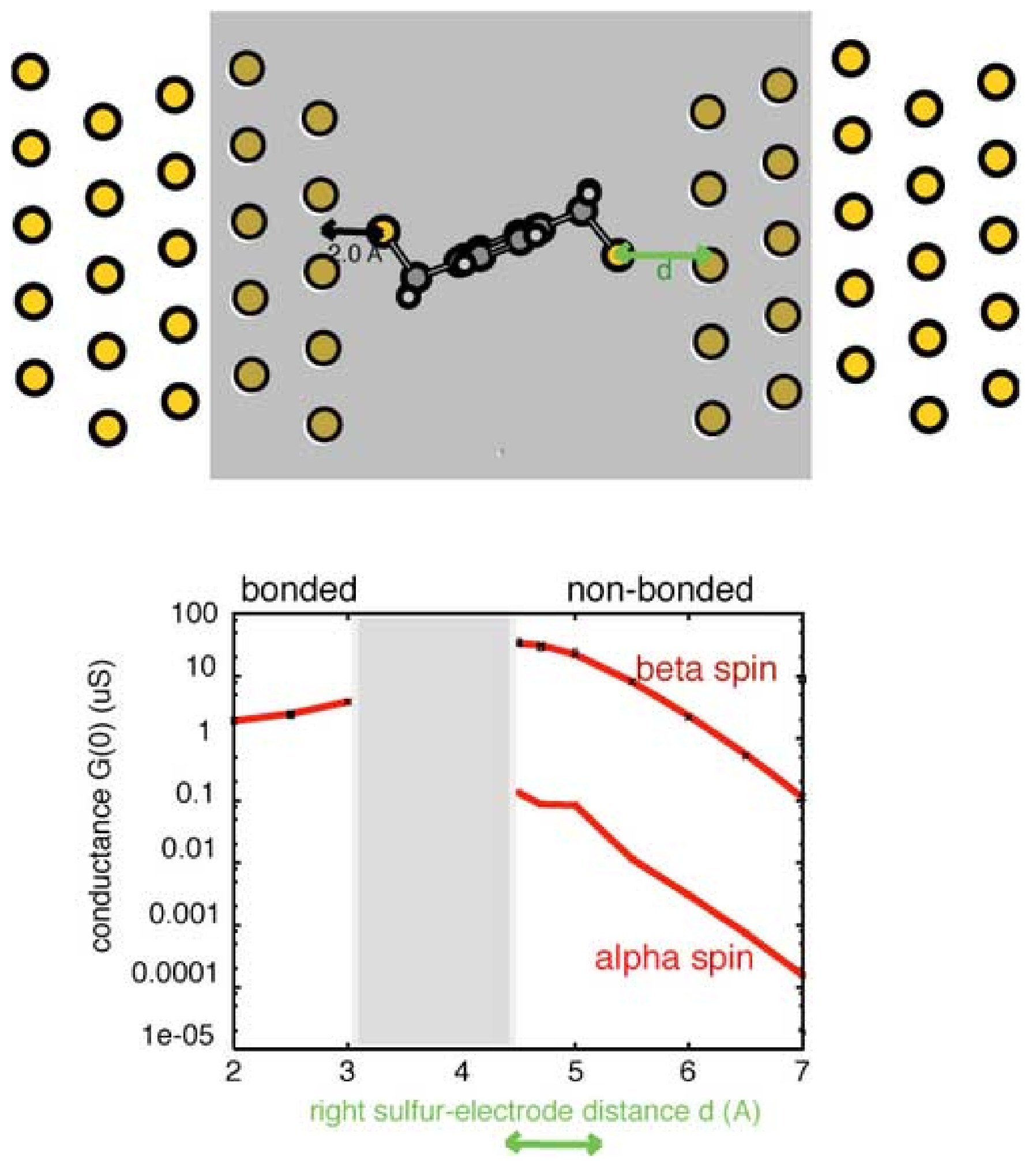


\section{* Graphical Abstract (synopsis)}

\section{Synopsis}

Density functional theory electronic structure and transport calculations reveal the surprising prediction that the tunneling current though a simple electrode-moleculeelectrode system can increase substantially when the sulphur gold bond anchoring the molecule to one electrode is stretched and even broken. 
Ms. No.: CPLETT-06-1031

Title: Prediction of Increased Tunneling Current by Bond Length Stretch in Molecular Break Junctions Corresponding Author: Prof Mike J Ford Authors: Rainer C Hoft, B.Sc. Hons; Michael B Cortie, Ph.D.

\section{Response to reviewer \#1 comments.}

We thank the reviewer for their critical reading of our manuscript and constructive comments. We have considered the comments very carefully and revised our manuscript accordingly. Each of the reviewers comments are addressed in the following, together with a description of the revisions made.

1. My main concern is that the result of this paper is rather thin. There have already been similar calculations in the literature on bond length effect to conductance. It has been well known that equilibrium bond length may or may not give the highest conductance. For tunneling through a molecule bridge, it is more important whether a resonance is set up or not. Changing bond length of the contact may help that effect. Therefore, as a reader I'd like to see more results on the contact details such as various surfaces, any correlation with work function of metal, more contact groups etc. S-Au has been studied extensively already. Also, it would improve the paper if information is provided as why the thiol-Au bond behaves different.

Yes, comments have been made in the literature before regarding change in conductance due to bond length change. However, the work we report is the first systematic study of this phenomenon. Guo et al (2005) point out that the current could go up or down upon moving away from the equilibrium bond length. Xue and Ratner (2005), and Ke et al (2005) calculate an increase in conductance with increasing molecule-electrode distance, however the calculations in both these cases are significantly less complete. Most importantly we have explicity taken into account the electron spin using a spin unrestricted calculation, and addressed directly the problem of bond dissociation. In our calculations we have determined over which bond-length regions the single-configuration calculation gives a reliable result. Previous calculations have not addressed this problem.

We have added comments in the introduction to highlight the originality of our work.

$\mathrm{S}$ on $\mathrm{Au}(111)$ has been chosen as a model system because the calculations can, in principle, be tested experimentally. Calculations of other surfaces or contact groups are less relevant therefore.

The following has been added to the conclusion to explain why the thiol-Au bond behaves differently: "The difference in behaviour for the thiol-Au and thiolate-Au bonds can be attributed to the strong chemisorption nature of the latter where electrons are shared between the $\mathrm{S}$ and surface $\mathrm{Au}$ atoms. It is the fate of the shared electron as the bond is broken that gives rise to the anomalous increase in conductance. The decrease in 
molecule-electrode coupling is, in this case, more than offset by the localization of the molecular orbitals close to the Fermi energy as the bond dissociates. By contrast the Authiol bond is a weak physisorbed bond with no electron sharing. Hence the conductance simply decreases as the bond is stretched because the orbitals do not significantly change."

2. The model in eq.1 is simply a tunneling formula. While the authors manage to use it (perhaps adding many similar terms) to fit their ATK numerical data in the figures, the entire discussion (page 7) did not really provide a physical picture why conductance can increase when $S$-Au bond is stretched. In other words, what's the reason the transmission peak moved the way it did.

The discussion of figure 3 has been modified to explain why the transmission peaks move the way they do. The following sentences have been added: "Orbital hybridization between the Au based orbitals and S based orbitals decreases as the bond is stretched. At small bond lengths the molecular $\mathrm{S}$ orbitals gain considerable character from the $\mathrm{Au}$ substrate orbitals giving a density of states which is spread over a large energy region. As the bond is stretched there is less mixing and the molecular S orbitals become more localized in energy leading to a much larger density of states concentrated over a much smaller energy window. This gives rise to the sharp peaks just below the Fermi level in Fig 3(b) at large bond distances which are due to orbitals of the right sulfur atom corresponding to the HOMO and LUMO orbitals of the isolated radical. This gives rise to the large , narrow peak in the transmission spectrum at large bond-lengths.."

3. In their fitting, how is interaction energy obtained? Using alpha $=0.1$, what values of coupling is obtained in unit of meV? Are these values reasonable?

Interactions energies are obtained directly from the ab initio calculations, this stated in the discussion of the model. Coupling of order $240 \mathrm{meV}$, at equilibrium bond length, is obtained. This is a reasonable value based upon comparison with values given in Datta. A sentence has been added to this effect.

\section{Why the alpha and beta spins behave so differently?}

For the thiolate bond there is a pair of electrons shared between the $\mathrm{S}$ atom and surface Au atoms. As the bond is dissociated one of these electrons remains on the S atom, and hence there is a single unpaired electron on the thiolate radical. There is now one alpha electron which is not paired with a beta electron, whereas all the beta electrons are paired. Hence the alpha and beta electrons behave differently.

The following sentence has been added to the discussion of Figure 4: "The alpha and beta electrons behave differently because there is a single unpaired alpha electron on the $\mathrm{S}$ atom as the bond is broken."

5. On the numerical calculations, very small number of $2 d k$-sampling is used for converging the density (2d BZ in the transverse direction). Data should be provided to 
show that this is enough. Did the authors check charge neutrality of their calculation in the transport (ATK) simulations? What's the quality of charge neutrality?

It was necessary to compromise between use of computational resources, and the accuracy of the k-point grid and orbital confinement parameters. Since a very large orbital cutoff is desirable (see point 6 below), we used a 3x3 k-points in the xy-plane (plane of the surface) for the ATK calculation (as opposed to $5 \times 5$ for the geometry optimization, which we know from our previous work, ref 18, is very well converged). The convergence of total energy and zero-volt conductance in ATK was tested as a function of the size of the k-point grid used (see Fig. 1 below). Here use of a less accurate orbital cutoff parameter allows us to do the calculation at much better k-point sampling. Note that the number of k-points $\mathrm{N}_{\mathrm{T}} \mathrm{XN}_{\mathrm{T}}$ used in calculating the transmission function can be different from the number of k-points $\mathrm{N}_{\mathrm{EXN}} \mathrm{N}_{\mathrm{E}}$ used in the electronic structure calculation, since the transmission function is calculated using the self-consistent electronic structure. From Fig 1, values of $\mathrm{N}_{\mathrm{E}}=2$ and $\mathrm{N}_{\mathrm{T}}=5$ give well-converged values. In the results presented in this work, we have used $\mathrm{N}_{\mathrm{E}}=3$ and $\mathrm{N}_{\mathrm{T}}=13$ which is well inside this convergence point. In the z-direction (perpendicular to the surface) we use 100 k-points both for the electronic structure and transmission parts of the ATK calculation. Again we have tested convergence of zero-volt conductance and total energy and suitable convergence is reached well below this point.

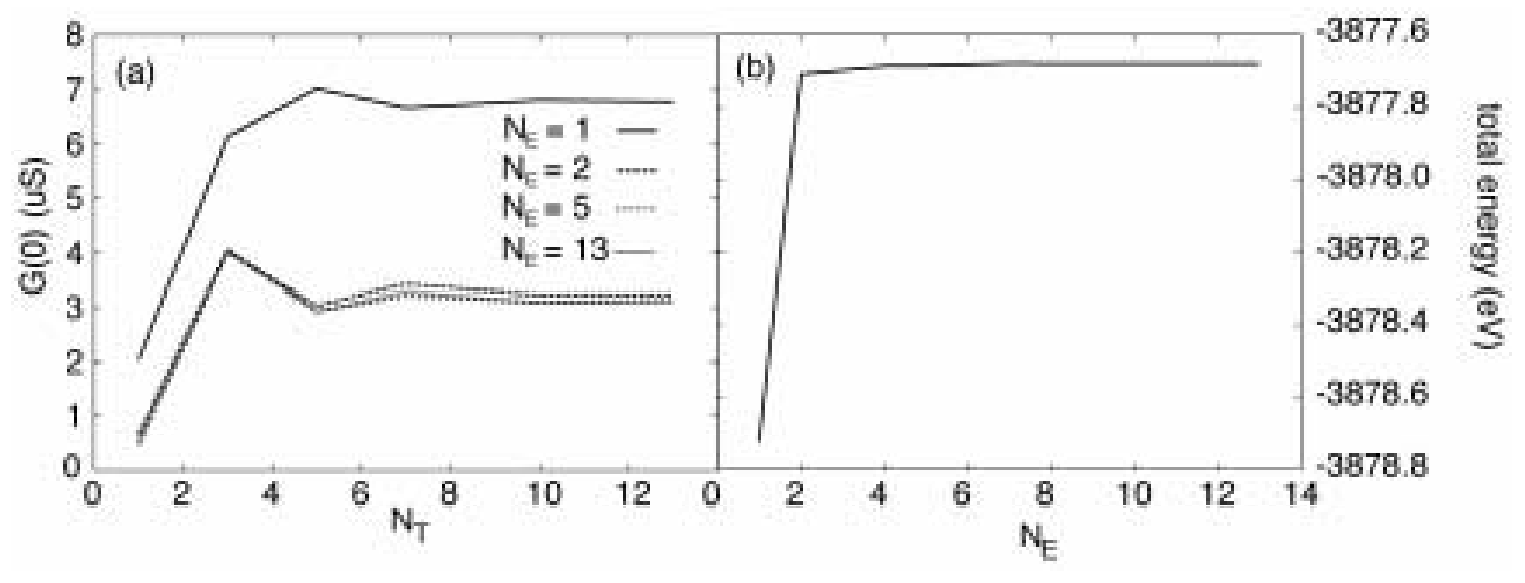

Fig. 1: (a) Zero-volt conductance of the $\mathrm{Au}(111)-\mathrm{XYL}-\mathrm{Au}(111)$ system as a function of the number of k-points used in the electronic structure and transmission spectrum calculations. (b) The total energy of the system as a function of the number of k-points used in the electronic structure calculation.

We have modified the methodology description to clarify these points. To conform to the length restrictions on the manuscript and to avoid disrupting the flow of the paper we have avoided including too much computational detail, such as the figure shown above.

The device region in the ATK calculations contains 2 layers of $3 \times 3$ gold atoms on either side of the molecule. The ionic charge in the device region is therefore $448(396+52)$ in the thiolate bonded case and $449(396+53)$ in the thiol bonded case. For the range of calculations presented here, the electronic charge reported by ATK in the device region is 
$448.05<$ Qe $<448.07$ and $449.06<$ Qe $<449.08$ in the thiolate and thiol bonded cases respectively. The deviation from charge neutrality is thus insignificant.

6. When $S$-Au bond is stretched to 5A, I imagine there is very little orbital overlap between the $S$ and the Au atoms. What are the orbital cutoffs in their siesta basis? If this orbital overlap is very small, I am worried about the accuracy of the calculations.

This is a very good point, a large orbital cut-off radius (i.e. small orbital confinement energy) is crucial to obtaining well-converged results. We have also tested this extensively in our previous work (ref 18) with respect to total and interaction energies and concluded that an energy-shift parameter of $5 \mathrm{mRy}$ (corresponding to the rise in orbital energy due to the confinement) gives well-converged interaction energies.

However, as the referee points out, in the present work the bond length is stretched and larger orbital radii may be needed. Consequently we have used an energy-shift parameter of $0.1 \mathrm{mRy}$ in the transport calculations. This corresponds to actual orbital radii of 4-5 A, for the various orbitals. Note that this means when atoms are $8 \mathrm{~A}$ apart, the orbital overlap will start being lost. Table 1 shows a test case where the zero-volt conductance with an Au-S distance d=7.0 A was calculated for different values of the energy-shift parameter. We conclude that $0.1 \mathrm{mRy}$ is a sufficiently accurate value to use in the present context.

\begin{tabular}{|l|l|l|}
\hline Energy shift parameter $(\mathrm{mRy})$ & $\mathrm{G}(0)$ spin alpha $(\mathrm{uS})$ & $\mathrm{G}(0)$ spin beta $(\mathrm{uS})$ \\
\hline 1.00 & 0.0000082 & 0.0040679 \\
\hline 0.10 & 0.0000788 & 0.0412114 \\
\hline 0.05 & 0.0000861 & 0.0488064 \\
\hline
\end{tabular}

Table 1: Zero-volt conductance for different energy shift parameter values. Au-S distance at right electrode is $7.0 \mathrm{~A}$.

Orbital confinement parameters (energy-shifts) are given in the methods section. Due to length restrictions we have not included a detailed discussion as given above.

In summary, while this referee thinks that understanding molecule-metal contact is a very important problem in molecular conduction, I am not sure this paper has adequately aided this field of work. I suggest the authors consider above points, revise the paper by adding more physics and results, and resubmit it to this journal.

We have revised our manuscript as recommended and described above. A number of other typographic errors have been corrected and minor re-wording made in order to meet the length requirement. We believe these results do contribute to the field of work because it is the first comprehensive exploration of this unexpected phenomenon. Our work takes into account the fact that single-configuration calculations fail to describe bond breaking under certain conditions. Using spin unrestricted calculations we can pinpoint exactly where the calculations fail. Previous calculations have not addressed this point. Our results are significant, original and timely, and merit publication as a letter. 


\title{
Prediction of Increased Tunneling Current by Bond Length Stretch in Molecular Break Junctions
}

\author{
R. C. Hoft, M. J. Ford*, and M. B. Cortie \\ Institute for Nanoscale Technology, University of Technology Sydney, PO Box 123, \\ Broadway NSW 2007, Australia
}

(Received )

We use ab-initio calculations of the tunneling current through a 1,4phenylenedimethanethiol (XYL) molecule adsorbed between $\mathrm{Au}(111)$ electrodes to show that there are circumstances under which tunneling currents can be increased by bond stretching. The effect is dependent upon the geometry of the gold-sulfur bond and does not occur for the physisorbed thiol, that is, a thiol with the hydrogen atom still in place. However, we predict that when the hydrogen atom is removed, causing the sulfur atom to be strongly bound to the gold surface, stretching this bond away from equilibrium will actually increase the tunneling current.

PACS numbers: 73.63.-b, 73.40.Gk, 85.35.-p

\footnotetext{
* Corresponding author details: mike.ford@uts.edu.au Institute for Nanoscale Technology University of Technology, Sydney PO Box 123, Broadway, NSW 2007 Ph: 61(0)2 95147956

Fax: 61(0)2 95148349
} 
Since theoretical prediction of current rectification in a single molecule by Aviram and Ratner in 1974 [1], molecular electronics has become an active area of research. Interest stems from the potential to exploit this transport phenomena to create electronic circuit components from individual molecules [2]. Despite impressive advances in experimental [3] and theoretical [4] techniques, there is often poor agreement between results for electron transport through individual molecules [5]. The most common first principles technique employed, namely density functional theory (DFT) [6, 7], is a theory designed for equilibrium situations and potential problems with its application to the nonequilibrium case have been pointed out by various authors $[8,9]$. Nevertheless, current understanding of the issues involved in these calculations will permit reliable predictions provided that it is understood that the conductances are acutely sensitive to experimental geometry, which is generally unknown. A statistically-based approach in which many possible geometries are sampled and averaged gives improved congruence with experimental data [10].

One of the most intriguing, recent theoretical predictions is increasing conductance when the tunneling distance between electrode and molecule is increased [10-12]. Here, we address this counter-intuitive phenomenon in detail. We study the model system, $\mathrm{Au}(111)-\mathrm{XYL}-\mathrm{Au}(111)$ and perform spin-polarised DFT calculations with the non-equilibrium Green's function (NEGF) formalism for calculating transport. XYL, or 1,4-phenylenedimethanethiol is a thiol-terminated aromatic molecule that easily selfassembles on a gold substrate [13]. We perform a thorough investigation of the effect of increasing the sulfur-gold distance, both with and without the presence of a terminal hydrogen atom. Crucially, spin-polarised calculations are used to identify regions, during 
bond-breaking, where single-configuration calculations fail due to spin-contamination. Previous DFT + NEGF studies of benzene-dithiol molecules sandwiched between gold electrodes have shown results that support our conclusions [11, 12], but do not appear to address this problem of bond-breaking. The results, therefore, contain some degree of uncertainty. In addition, there are differences in the computational approach used. In Ref. 11 six Au electrode atoms are included in the "extended molecule" and treated using DFT while the rest of the semi-infinite electrodes are treated using a tight-binding approach. In Ref. $12 \mathrm{Au}(100)$ leads with finite cross-section are used. Here, we treat the electrodes as semi-infinite $\mathrm{Au}(111)$ surfaces, and treat the entire system on the same footing using DFT (see Fig. 1). Our results demonstrate that this effect is not isolated to one molecule and one surface, and more importantly is not an artifact caused by the inability of DFT to describe bond dissociation correctly.

It is generally assumed that the sulfur-hydrogen bond cleaves and a strong chemical bond forms when thiol molecules interact with a gold surface. There is considerable evidence to support this formation of a thiolate bond [14]. A weaker, physisorbed bond is possible without removal of the hydrogen. Both bond configurations are investigated here. A thiolate bond, with equilibrium bond length, is kept between left $\mathrm{Au}(111)$ electrode and molecule, whereas we interchange the geometry on the right electrode between thiolate (geometry A) and thiol (geometry B) and vary the sulfur to surface distance $d$ (Fig 1).

Optimum adsorption geometries were calculated with the SIESTA software package [15], a density functional code employing a linear combination of numerical atomic orbitals as basis functions. Double zeta plus polarization orbitals are used for the valence 
electrons with pseudo-potentials for the core electrons and nucleus Orbitals are strictly localized, a single energy confinement parameter determines the cut-off radius. Here we set this parameter to $5 \mathrm{mRy}$. The local density approximation (LDA) to the exchangecorrelation functional is used, incorporating the self-interaction correction by Perdew and Zunger [16]. Calculations are spin-polarised with the (111) surface represented by a $3 \times 3$ unit cell, 4 layers thick. A 5x5 k-point Monkhorst and Pack [17] grid is used in the plane of the gold surface. The surface layer is not relaxed during optimization. We have previously examined the reliability of this type of calculation against the computational conditions used [18], the present conditions give very well converged optimum geometries.

This relaxed geometry is then used in the transport calculation conducted with the ATK package [19]. This uses the SIESTA method to obtain electronic structures and the NEGF formalism to calculate transport. The molecule with two layers of Au atoms on either side is modeled as the device region, connected on the left and right by semi-infinite electrodes (Fig. 1). Here, single zeta (double-zeta) plus polarization orbitals are used for electrode (molecule) atoms. A 3x3 k-point grid in the plane of the surface and $100 \mathrm{k}$ points in the perpendicular direction are used for the electronic structure part of the calculation, and a $13 \times 13 \times 100$ k-point grid for the transport calculation. The orbital confinement is reduced to $0.1 \mathrm{mRy}$ so that it does not affect the calculation. [20]. Extensive tests were performed to check convergence against the k-point grid and orbital cut-off radius. The zero-volt conductance and total energy do not change if both these parameters are increased. We have also tested the effect of re-optimisation of the 
geometry as the bond-length is increased, at $3 \AA$ the change in tunneling current upon relaxation is negligible.

For the gold-thiolate bond, the preferred adsorption site is between the bridge and fcchollow sites, $2.0 \AA$ above the surface. For the gold-thiol bond it is between the on-top and hcp-hollow sites at a height of $2.4 \AA$. In both cases the total spin is zero. The calculations use a $25 \mathrm{meV}$ Fermi smearing temperature to aid convergence and allow partial occupation of states. Therefore the total spin is zero state in both systems, even though they differ by one electron.

Fig. 2(a) shows the ab-initio zero volt conductance for $\alpha$ - and $\beta$-spin electrons for geometry A (see Fig. 1) as function of sulfur-electrode distance. The shaded region indicates where the system is not in a pure spin state, i.e. where spin-contamination occurs. For this region more sophisticated multi-configurational methods need to be used. To the left of the shaded region $(d \leq 3.0 \AA)$ the XYL radical is bound to the gold and the overall spin is zero (singlet state). To the right ( $d \geq 4.5 \AA$ ) the sulfur-gold bond has been broken and there is an unpaired electron on the sulfur atom, it is a doublet state. In these two regions the present single-configuration calculations are reliable. Counter-intuitively, the conductance rises as the bond is stretched to $3.0 \AA$, in confirmation of observations made by others using somewhat different computational techniques [11, 12]. Furthermore the conductance for $\beta$-spin electrons continues to increase substantially after the bond has been broken up to a distance of $5.0 \AA \AA$ before decaying to zero at large $d$. For $\alpha$-spin electrons, the conductance decays after the bond is broken. Note that there is an unpaired spin- $\alpha$ electron on the sulphur atom. The same trend is obtained for the tunneling current at a low bias of $0.1 \mathrm{~V}$ (not shown). 
In geometry B the system remains in a spin singlet state as $d$ is increased as there is no longer an unpaired electron. Fig. 2(b) shows the exponential decay in $0 \mathrm{~V}$ conductance for $\alpha$ - and $\beta$-spin electrons (identical in this case) as a function of sulfur-surface distance.

The increase in conductance for the thiolate molecule can be attributed to a large, narrow peak in the transmission spectrum for $\beta$-spin electrons that develops as the sulfurgold bond is stretched, Fig 3(a). The origin of this becomes apparent from the density of states projected onto the entire molecule (PDOS) as shown in Fig 3(b). Orbital hybridization between the Au based orbitals and S based orbitals decreases as the bond is stretched. At small bond lengths the molecular S orbitals gain considerable character from the Au substrate orbitals giving a density of states which is spread over a large energy region. As the bond is stretched there is less mixing and the molecular $\mathrm{S}$ orbitals become more localized in energy leading to a much larger density of states concentrated over a much smaller energy window. This gives rise to the sharp peaks just below the Fermi level in Fig 3(b) at large bond distances which are due to orbitals of the right sulfur atom corresponding to the HOMO and LUMO orbitals of the isolated radical. This gives rise to the large, narrow peak in the transmission spectrum at large bond-lengths.

Fig. 4 (a) shows quantitatively how the orbital-eigenvalues of the $\beta$-spin electrons align with the Fermi level as the bond is stretched. It is initially surprising that this change in the HOMO and LUMO orbitals is able to overcome the exponentially decreasing coupling between the molecule and the right electrode (as shown by the interaction energy in Fig 4(b)) and give an increased conductance. However, the phenomenon can be understood with the aid of a simple one-level model to obtain a qualitative picture of the effect of the 
change in orbital eigenvalues and coupling on the conductance. The current through a channel with a single level with energy $\varepsilon$ between two reservoirs is given by [21]

$$
i(V)=\frac{e}{h} \gamma_{1} \gamma_{2} \int_{-e V / 2}^{e V / 2} d E\left[(E-\varepsilon)^{2}+\gamma^{2}\right]^{-1}
$$

where we have used the zero-temperature approximation and assumed a Lorentzian density of states. $\gamma_{1}$ and $\gamma_{2}$ are the coupling strengths of the level to the left and right reservoirs, $\gamma=\left(\gamma_{1}+\gamma_{2}\right) / 2 . E$ is the energy relative to the Fermi level. Equation (1) can readily be integrated to find the current or differentiated to find the conductance as a function of voltage.

In this model it is assumed that the coupling strength of the energy level to both electrodes is the same and proportional to $\Gamma$, the interaction energy of the molecule with the right electrode, i.e. $\gamma_{1}=\gamma_{2}=\gamma=\alpha \Gamma$ for some constant $\alpha$. This will have the right qualitative behavior since an increase in $d$ causes the two orbitals we are considering to become less hybridized with the electrode orbitals. The coupling to both electrodes thus decays exponentially with an increasing distance, as does the interaction energy with the right electrode. We then calculate the total current and conductance as the sum of the one-level values calculated from equation (1) for the HOMO and LUMO orbitals, using the ab-initio eigenvalues and interaction energies from Fig 4. The results are plotted in Fig 2 . The only empirical parameter is $\alpha$ which was set to 0.1 in order to obtain the best quantitative agreement between the one-level model and ab-initio results in the thiolate bonded case. The qualitative behavior does not change if $\alpha$ is varied. This value of $\alpha$ gives a coupling energy of about $240 \mathrm{meV}$ at equilibrium bond length, a value which is reasonable compared with values given by Datta [21]. 
In summary, we have performed ab-initio calculations of the zero volt conductance for the 1,4-phenylenedimethanethiol molecule absorbed between two gold electrodes as the sulfur-surface distance on one electrode is increased. Contrary to expectation, the maximum tunneling current for the thiolate molecule does occur not at the equilibrium adsorption geometry. Rather, we predict the tunneling current increases as the sulfur-gold bond is stretched and continues to increase after the bond is broken, before eventually decaying to zero. For the thiol molecule we predict the expected exponential decay in tunneling current with increased sulfur-electrode distance. The difference in behaviour for the thiol-Au and thiolate-Au bonds can be attributed to the strong chemisorption nature of the latter where electrons are shared between the $\mathrm{S}$ and surface Au atoms. It is the fate of the shared electron as the bond is broken that gives rise to the anomalous increase in conductance. The decrease in molecule-electrode coupling is, in this case, more than offset by localization of the molecular orbitals close to the Fermi energy as the bond dissociates. By contrast the Au-thiol bond is a weak physisorbed bond with no electron sharing, and the conductance decreases as the bond is stretched because the orbitals do not significantly change.

We believe the results presented here are a sound theoretical prediction which is worth testing experimentally. They are counterintuitive and occur in a widely studied system, and hence have potentially far reaching consequences in the field of molecular electronics.

This work was supported by The University of Technology, Sydney. Computing facilities were provided by the Australian Centre for Advanced Computing and Communication 
(New South Wales) and the National Facility at the Australian Partnership for Advanced Computing.

[1] A. Aviram and M. A. Ratner, Chem. Phys. Lett. 29 (1974) 277.

[2] M. A. Reed, Proc. Ieee 87 (1999) 652.

[3] D. K. James and J. M. Tour, Chem. Mater. 16 (2004) 4423.

[4] Y. Q. Xue and M. A. Ratner, Int. J. Quantum Chem. 102 (2005) 911.

[5] A. Nitzan and M. A. Ratner, Science 300 (2003) 1384.

[6] P. Hohenberg and W. Kohn, Phys. Rev. 136 (1964) B864.

[7] L. J. Sham and W. Kohn, Phys. Rev. 145 (1966) 561.

[8] G. C. Solomon, J. R. Reimers, and N. S. Hush, J. Chem. Phys. 121 (2004) 6615.

[9] P. S. Krstic, D. J. Dean, X. G. Zhang, D. Keffer, Y. S. Leng, P. T. Cummings, and J. C. Wells, Comp. Mater. Sci. 28 (2003) 321.

[10] Y. B. Hu, Y. Zhu, H. J. Gao, and H. Guo, Phys. Rev. Lett. 95 (2005) 156803.

[11] Y. Q. Xue and M. A. Ratner, Phys. Rev. B 68 (2003) 115407.

[12] S. H. Ke, H. U. Baranger, and W. T. Yang, J. Chem. Phys. 122 (2005) 074704.

[13] J. I. Henderson, S. Feng, G. M. Ferrence, T. Bein, and C. P. Kubiak, Inorg. Chim. Acta 242 (1996) 115.

[14] J. C. Love, L. A. Estroff, J. K. Kriebel, R. G. Nuzzo, and G. M. Whitesides, Chem. Rev. 105 (2005) 1103.

[15] J. M. Soler, E. Artacho, J. D. Gale, A. Garcia, J. Junquera, P. Ordejon, and D. Sanchez-Portal, J. Phys.-Condens. Mat. 14 (2002) 2745.

[16] J. P. Perdew and A. Zunger, Phys. Rev. B 23 (1981) 5048. 
[17] H. J. Monkhorst and J. D. Pack, Phys. Rev. B 13 (1976) 5188.

[18] M. J. Ford, R. C. Hoft, and A. McDonagh, J. Phys. Chem. B 109 (2005) 20387.

[19] M. Brandbyge, J. L. Mozos, P. Ordejon, J. Taylor, and K. Stokbro, Phys. Rev. B 65 (2002) 165401.

[20] R. Rurali, N. Lorente, and P. Ordejon, Phys. Rev. Lett. 95 (2005) 209601.

[21] S. Datta, Quantum Transport: Atom to Transistor, Cambridge University Press, Cambridge, 2005. 


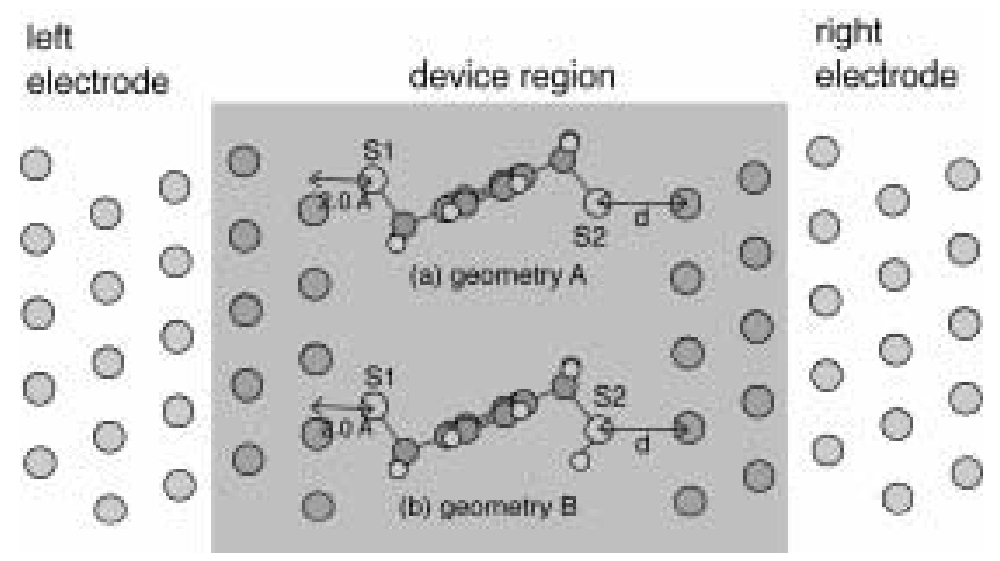

FIG 1. Bonding configurations 


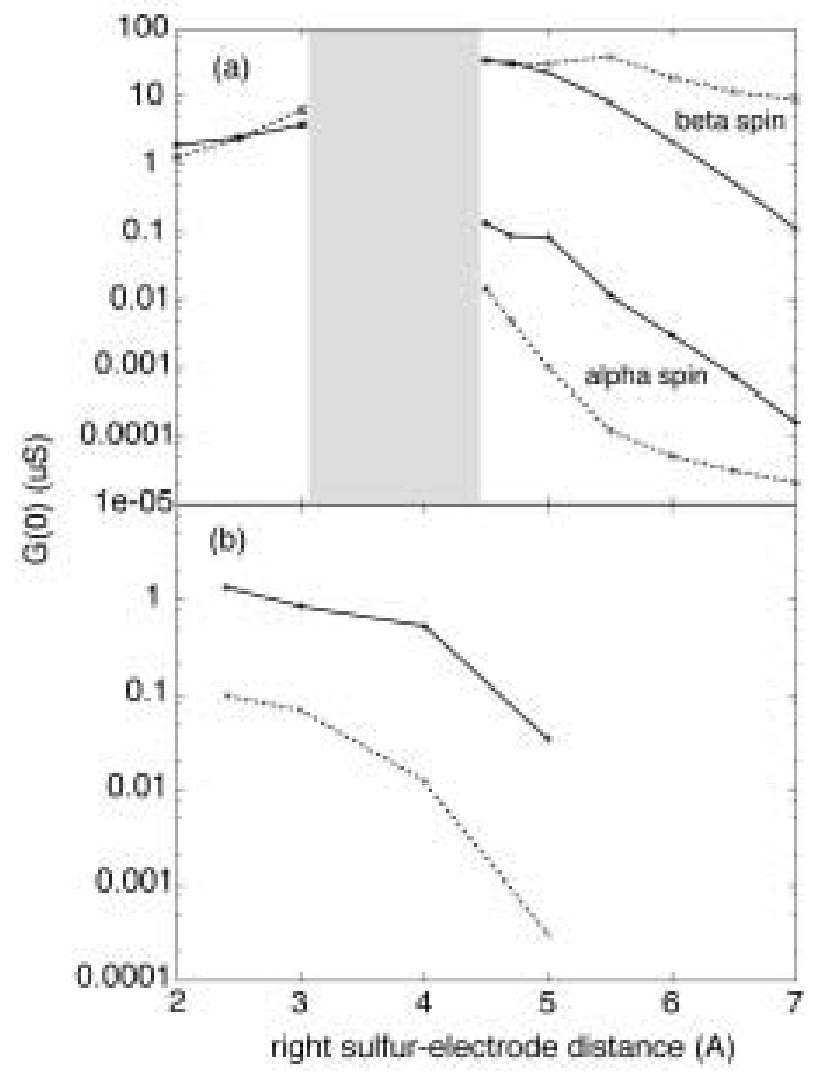

FIG 2. Zero volt conductance (a) geometry A and (b) geometry B as a function of sulfurgold surface distance. Solid lines are ab-initio calculations, dashed lines are a qualitative one-level model. 


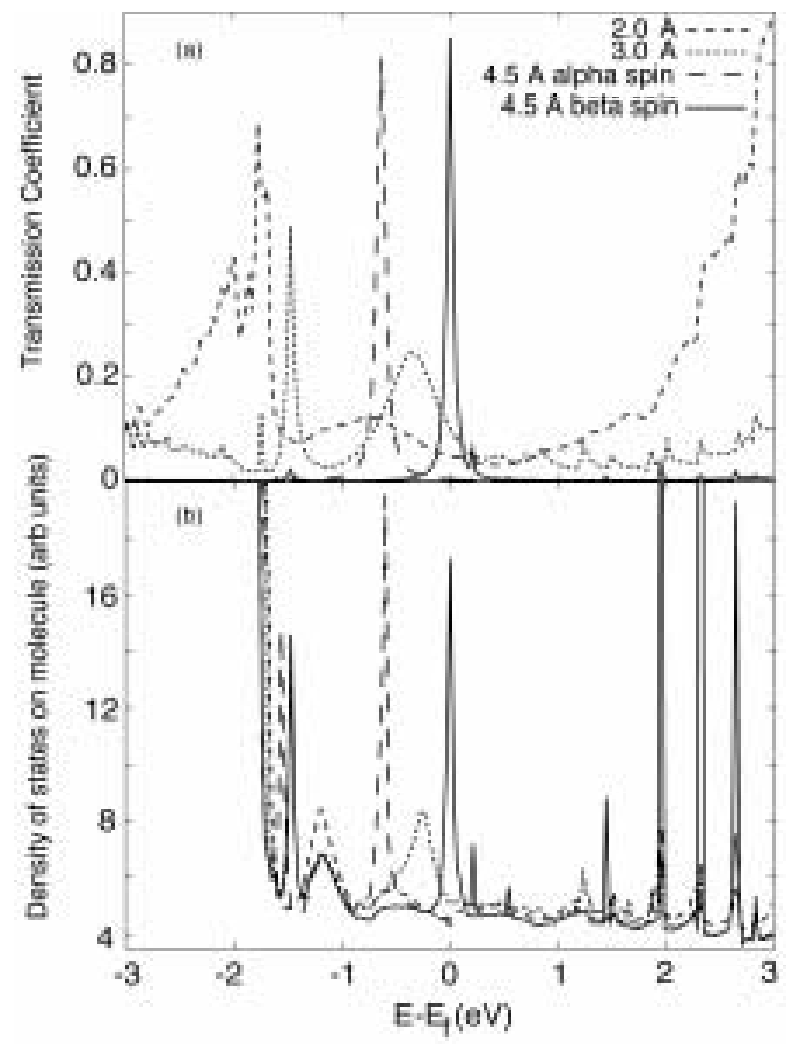

FIG 3. (a) Transmission coefficient and (b) density of states projected onto the molecule for sulfur-electrode distances, $d=2.0 \AA, 3.0 \AA$ and $4.5 \AA$ in geometry A. 


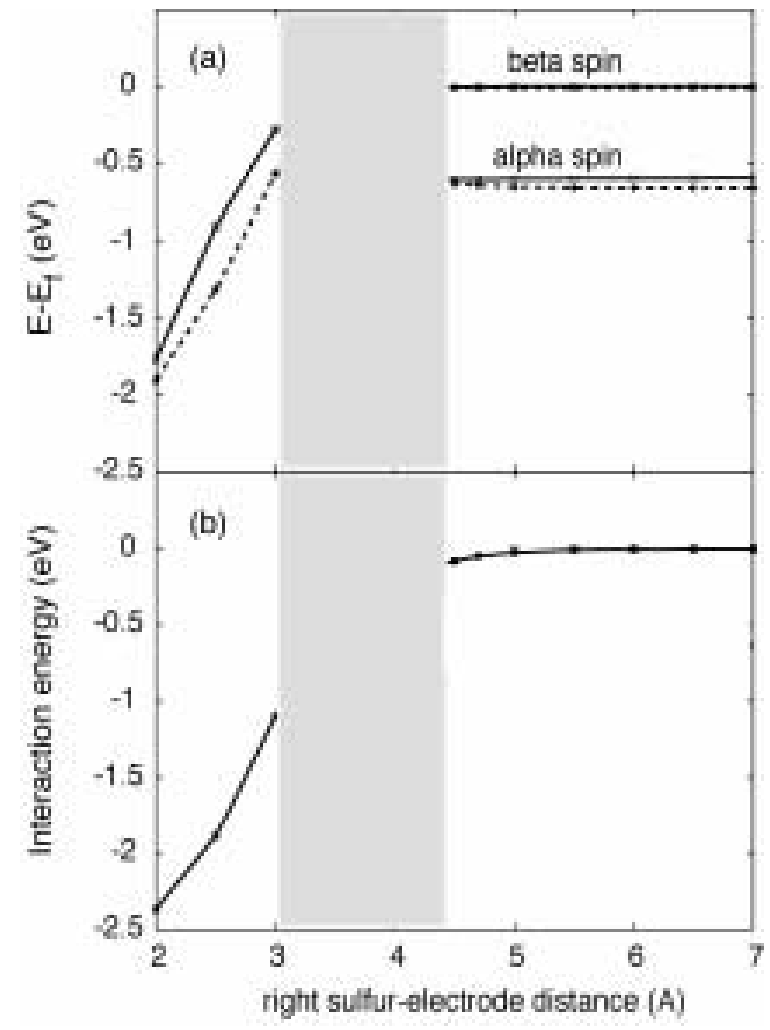

FIG. 4: (a) HOMO (dashed lines) and LUMO (solid lines) eigenvalues for spin- $\alpha$ and spin$\beta$ electrons and (b) molecule-surface interaction energy as a function of sulfur-gold distance for geometry A. 


\section{CHEMICAL PHYSICS LETTERS}

\section{Online Manuscript Submission Item Count}

\begin{tabular}{l}
\hline Manuscript Title: \\
Prediction of Increased Tunneling Current by Bond Length Stretch in Molecular Break \\
\hline Aunctions \\
\hline R. C. Hoft, M. J. Ford*, and M. B. Cortie
\end{tabular}

Please include a count of the following items. When you are finished, you may save this document for use with your online submission to Chemical Physics Letters.

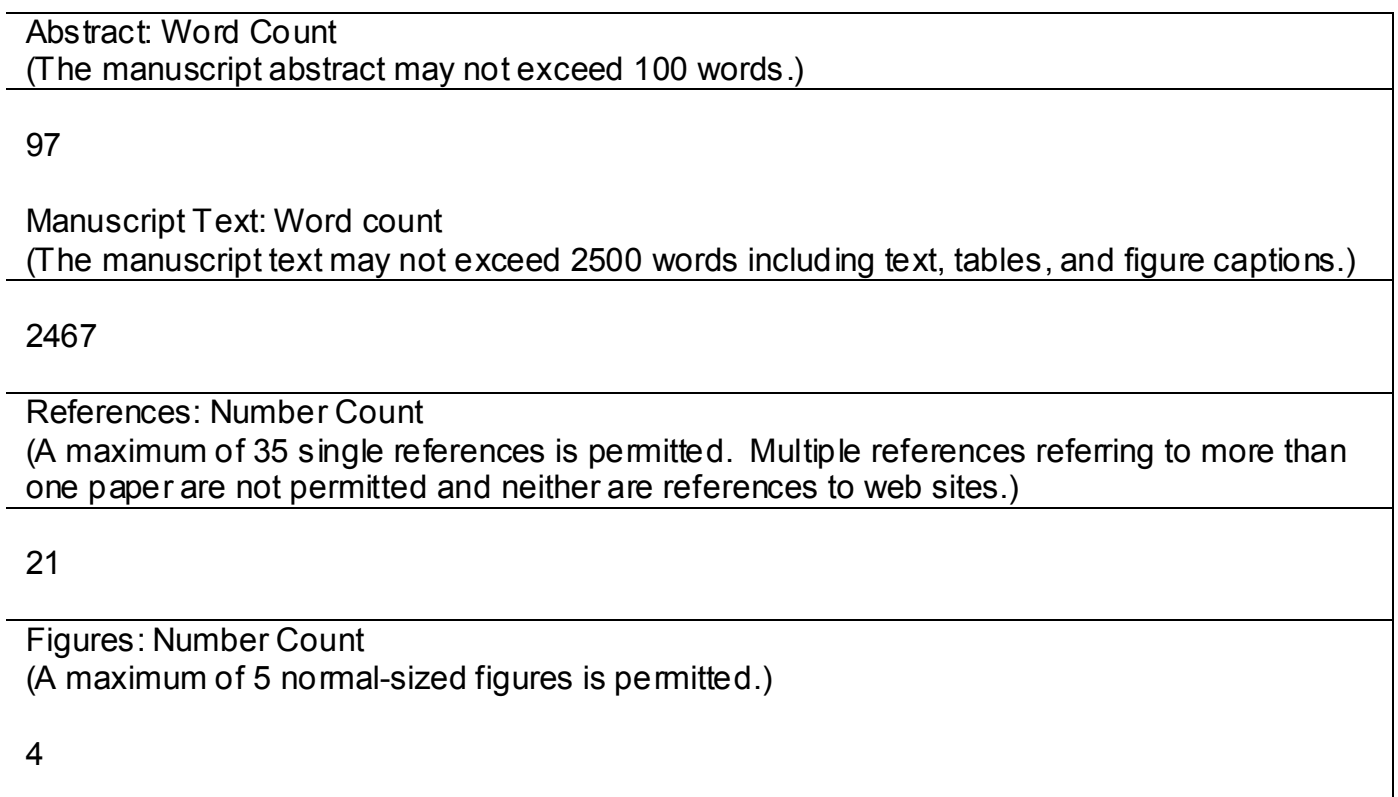

\footnotetext{
* Corresponding author details:

mike.ford@uts .edu.au

Institute for Nanoscale Technology

University of Technology, Sydney

PO Box 123, Broadway, NSW 2007

Ph: 61(0)2 95147956

Fax: 61(0)2 95148349
} 